\title{
REGISTRATION OF NUTRACEUTICALS AND REPORTING ADVERSE EVENTS OF DIETARY SUPPLEMENTS IN USA
}

Navya BR ${ }^{*}$, Dr. Shanthakumar GS, Shrikanth P, Ayushi Agrawal, Shoukat Ali.

Department of Pharmaceutical Regulatory Affairs, Acharya \& BM Reddy College of Pharmacy, Soladevanahalli, Acharya Post, Hesaraghatta Main Road, Bengaluru-560107.

Article Info: Received 03 May 2020; Accepted 02 June 2020

DOI: https://doi.org/10.32553/jbpr.v9i4.794

Corresponding author: Navya BR

Conflict of interest: No conflict of interest.

\section{Abstract}

The US FDA characterizes Nutraceuticals as "Dietary Supplements" Under DSHEA (Dietary Supplement Health Education Act-1994). In USA Food and Drug Administration (FDA) controls nutraceutical under different guidelines. According to DSHEA producer should make sure that nutraceutical is safe before it is promoted. The FDA concentrating on quality and safety of the product as these are used by humans. As food items are coming to starting with one nation then onto the next, keeping up safety and quality principles according to different regulatory frameworks set by the individual governments gets significant.

Key words: FDA, dietary supplements, quality, DSHEA, Nutraceuticals.

\section{INTRODUCTION}

Nutraceuticals, characterized as any part of food or food which gives medical advantages including prevention or treatment of sickness have emerged as a necessity for consumers in developed as well as developing countries. Dietary supplement (DS): Any food and food ingredient that may give medical advantage beyond the conventional nourishment than it contains.

The term "nutraceutical" was coined from "nutrition" and "pharmaceutical" in 1989 by Stephen Defelice, MD, founder and chairman of the Foundation for Innovation in Medicine (FIM), Cranford, NJ. According to Defelice, nutraceutical can be defined as, "a food (or part of a food) that provides medical or health benefits, including the prevention and/or treatment of a disease [1]. Different definitions and regulations are given for nutraceuticals in over the years, keeping the safety of people in mind [2].

Examination in the region of food science is giving foundation to advancement of such items. Such items have unique medical advantage claims or numerous multiple times claims for remedy for specific infections or issues. Guidelines in the region of such items change from nation to nation and numerous items have been propelled in showcase [3]. The understanding of regulatory framework of nutraceuticals will help to develop new regulations. In the current situation there are distinctive regulatory systems all over the world. Plants are one of the most significant assets of human nourishments and meds. Quickly expanding information on nourishment, medication, and plant biotechnology has radically changed the thoughts regarding horticulture, prosperity and food, got conflict on them [4].

\section{Dietary Supplements (DS)}

Based on certain criteria DSHEA has definite DS is an item that is anticipated to enhance the eating routine that contains at least one or more of the nutritional ingredient an botanical or other herbal, an amino acid, a mineral, a vitamin. These are only the enhancements which are included in the diet bringing about increment in the food admission of an individual per day these might be accessible as extracted solution, mixture of liquids, metabolic constituent, concentrates or any mix of the dietary parts [5].

Dietary supplements also found in various pharmaceutical dosage forms, such as Tablets, Capsules, Soft gels, Gel caps, Liquids, Powders etc. DS can be marketed without getting approval from USFDA. But efficacy, safety tests must be done by the FDA and proper labeling should be there on the product packaging. Americans including women, men and also children are falling debilitated as these DS were promising to give a superior \& enthusiastic life. These DS were expended as juice, food, and different supplements. Also, these are made a tempest in the government has it neglected to keep.

\section{Regulation of Dietary Supplements in US}

FDA directs dietary products and dietary ingredients under various guidelines managed by Dietary Supplement Health and Education Act (DSHEA):

- Producers and wholesalers of DS \& dietary ingredients are prohibited from promoting products that are misbranded or contaminated. This implies that these organizations are responsible for testing the labeling \& 
safety of the product before marketing them. They should meet all the prerequisites of DSHEA and FDA guidelines.

FDA can make the decision against any defiled or misbranded DS after it releases to the market.

The labeling of DS should contain the fallowing specific information:

(1) Net content of ingredients;

(2) Identity statement;

(3) Panel of the supplement Facts;

(4) Manufacturer or distributor name and address;

(5) Usage directions.

(6) Wherever necessary other Ingredients. Customs and Border Patrol requires that products, including DSs, comply with country of origin markings

Dietary supplement organizations are considered as food offices and may require FDA approval under the Bioterrorism Act. FDA additionally necessitates that the ingredients in a DS be allowed for use in the dietary enhancement either through exclusion or through another dietary ingredient application.

Any structure/work claim on the naming of a DS should be accounted to FDA inside 30 days of acquainting the DS to market. What are the factors to be considered in labeling versus marketing material is addressed through various court cases. Each circumstance is different, but it has been ruled that if a marketing material accompanies the product, even if it is not attached to the product, that marketing material may be considered as labeling and thus subject to FDA jurisdiction.

USFDA take care the safety of dietary supplements and its approval is not required

- Before manufacturing or selling their item, DS makers don't need to enroll with FDA, or acquire FDA approval,

- Before selling an item, producers are responsible for guaranteeing that a DS is safe. FDA has the position to make a move against risky DS items.

- Makers must guarantee that their labeling of DS product is honest and not ambiguous [6].

\section{Dietary Supplements (DS) Registration in US}

In US only those DS are registered, which should meet definition requirements given by the DSHEA. The 21 CFR 190 deals with the regulation of active ingredient of DS. During registration, it must be noted that whether it is an Active ingredient or Inactive ingredient

During the registration time, it must be taken into consideration that whether it is ODI or NDI. On the off chance that a DS is advertised before $15^{\text {th }}$ October 1994 it is considered as Old Dietary Ingredient (ODI). In the event that it is after the date, at that point it is New Dietary
Ingredient (NDI). Center for Food Safety and Applied Nutrition (CFSAN) carry out the PMS for the safety of the DS in the US market related to NDI products [7].

All the required documents for registration are submitted to

\section{Office of Nutritional Products,}

Labeling and Dietary Supplements (HFS-820), CFSAN, FDA, 5100 Paint Branch Pkwy, College Park, MD 20740.

The documents to be submitted are as follows:

1. Name and address of applicant

2. Name of product even the botanical name can be included.

3. New Dietary Ingredient or ODI type of dietary supplement stating statement.

4. Safety measures evidences if any

5. Distributor \& manufacturer signature.

The USFDA has given GMP guidelines for the Dietary supplements which are applied to all the foreign organizations and domestic organizations. This GMP guideline indicates the necessities for Manufacture, Package, label of dietary supplement and Regulatory prerequisites for registration [8].

\section{Health Claims in USA:}

There are three types of Health claims which are as follows:

1. Health claims: This was approved under the NLEA in the year 1990. These depict a connection between a food, food segment, DS ingredient and diminishing danger of disease.

Health claims can further classified into:

- Significant Scientific Agreement (SSA) Claims: These claims utilized for regular nourishments \& dietary supplements.

- FDA Modernization Act (FDAMA) Claims: These claims only utilized distinctly for regular nourishments \& can't be utilized on dietary supplements

- Qualified Health Claims: These claims can be utilized for regular nourishments \& dietary enhancements. Any manufacturer may request FDA to give a guideline with respect to a health claim (21 CFR 101.70). FDA assesses the appeal as indicated by the SSA standard.

2. Nutrient Content Claims: Some case is on substance of specific supplements / substances in a food, for example, decreased fat content \& are utilized to portray the level of that supplement in an item comparative with the everyday esteem. 
3. Structure/Function Claims: These were approved under the DSHEA 1994. Such cases identify with a positive commitment to health or to the improvement of a capacity or to changing or safeguarding wellbeing [9].

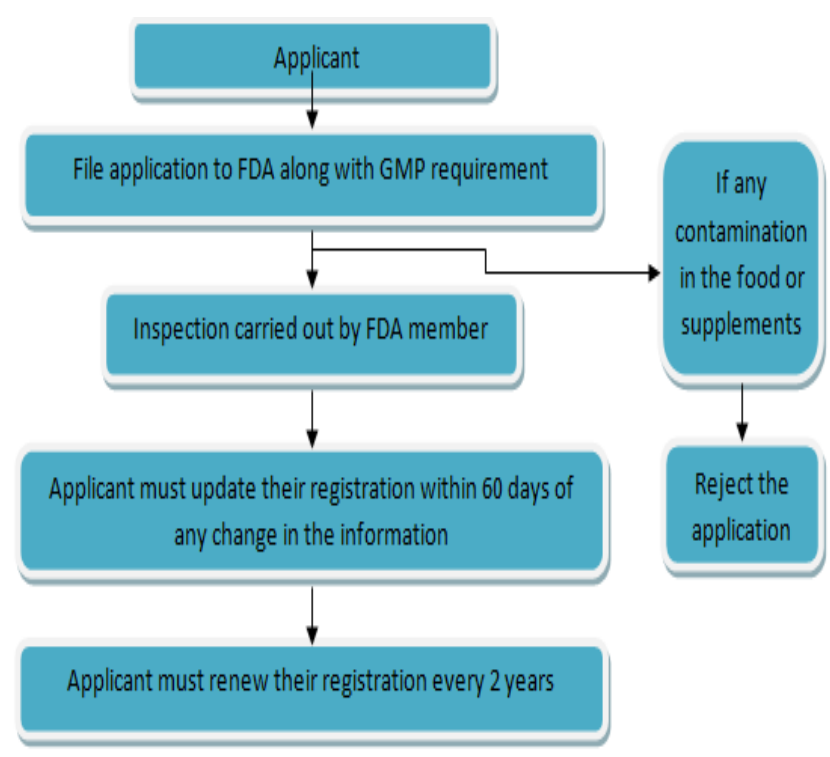

Fig.1: Registration Process of Nutraceuticals for US Market

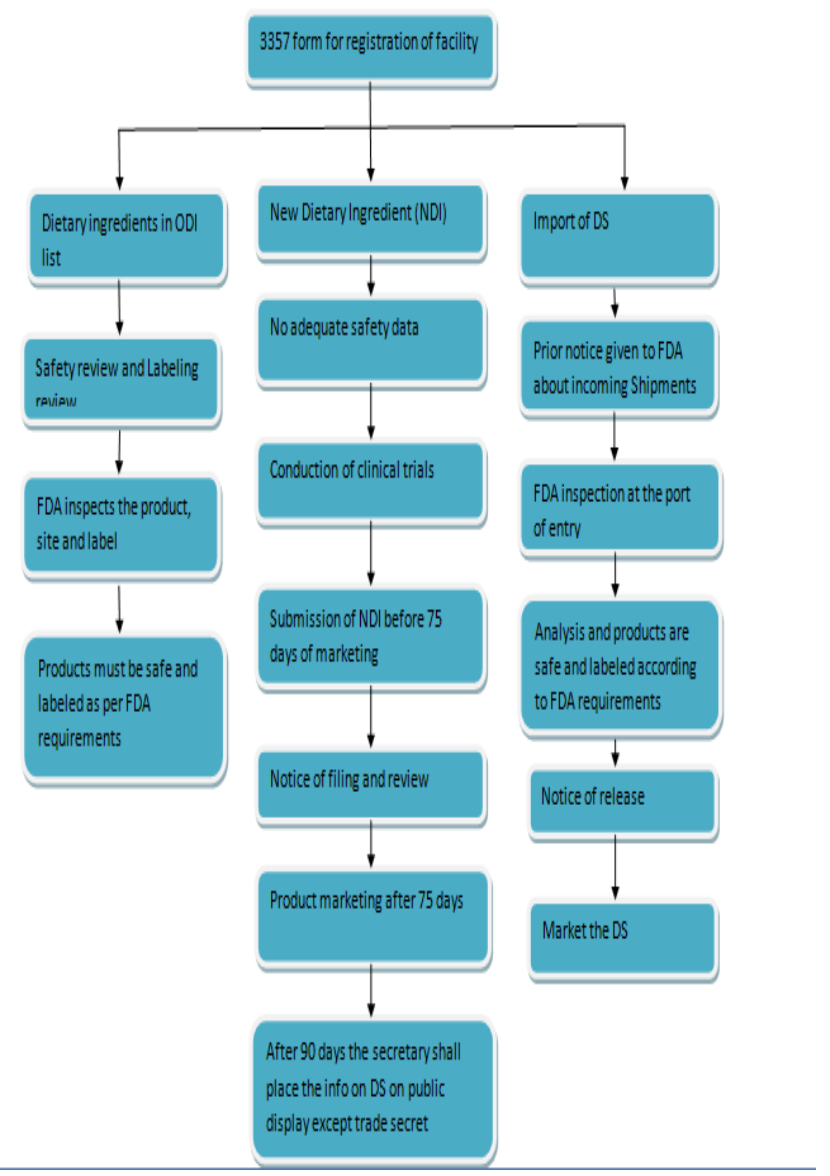

Fig.2: Regulatory Process for the Clearance [10]

\section{Reporting of Adverse Events of Dietary Supplements in USA}

FDA takes care of both completed DS product and dietary ingredients. FDA manages DS under an alternate arrangement of guidelines than those covering "regular" nourishments \& medication items. Under the DSHEA 1994:

- Manufactures \& merchants of DS \& dietary ingredients are denied from publicizing things that are tainted or adulterated. This infers that these associations are at risk for surveying the labeling \& safety of their things before elevating to ensure that they meet all the necessities of DSHEA and FDA rules.

- FDA can take action against any corrupted or adulterated dietary supplement.

- Dietary supplements include minerals, herbs, vitamins, amino acids, weight loss pills whey protein.

\section{Adverse Effects or Illness May Include:}

$\checkmark$ Blood in urine, stool, sputum or vomit

$\checkmark$ Mood changes, psychological, or social changes

$\checkmark$ Exhaustion, craving loss, yellowing skin/eyes

$\checkmark$ Extreme muscle/ joint pain

$\checkmark$ Slurred speech, uneven shortcoming of face, vision, arm, leg (stroke)

$\checkmark$ Abnormal bleeding from gums / nose

$\checkmark$ Rash, lip/throat /tongue expanding, wheezing, Itching

$\checkmark$ Low BP, blacking out, windedness, chest pain, palpitations, unpredictable heart beat

$\checkmark$ Extreme, steady sickness, diarrhea or stomach pain

$\checkmark$ Trouble in urinating, diminished urination

\section{For Consumers and Health Professionals:}

In case any consumer feels unhealthy or illness due to consumption of dietary supplement he/she can report problem in the Safety Reporting Portal to present patient objection to FDA.

Subsequent to signing in, select the choice "Start another report" \& pick "Dietary Supplement Report (voluntary)" Please transfer complete information. Complete reports are generally useful, yet even snippets of data can assist FDA with distinguishing possibly risky items.

\section{For Industry:}

Individuals from the DS industry may now utilize the announcing structure on the Safety Reporting Portal to meet the detailing necessities set up in segment 761 of the FD \& C Act. Subsequent to signing in, select the alternative "Start another report" \& pick "Dietary Supplement Report (required)".

\section{If Customer Need Assistance:}

In case of any queries regarding reporting on DS, please customers should contact DSR Support@fda.hhs.gov. 
While submitting safety report; customer can contact SRP Support@fda.hhs.gov for technical support [11].

\section{CONCLUSION:}

The Nutraceutical business is a developing area in emerging markets. The USA has changed the DSHEA in 1994 gives guidance for the registering of the Nutraceuticals in the nation for marketing purpose. Approval of dietary supplements doesn't come under FDA. FDA gives regulation for registering, labeling, GMP requirements for the marketing the dietary supplement. Reporting of adverse reactions occurred due to use of dietary supplements in the FDA safety portal help in reducing the risk and giving safe dietary supplement to customers.

\section{REFERENCES:}

1. Brower V. Nutraceuticals: Poised for a healthy slice of the healthcare market. Nat Biotechnol 1998; 16:728-31.

2. Zeisel SH. Regulation of Nutraceuticals. Science 1999; 285:185-86.
3. Palthur MP, Palthur SS, Chitta SK. Nutraceuticals: A conceptual definition. Int J Pharm Pharm Sci 2010; 2(3): 19-27.

4. Bagchi D. Nutraceuticals and functional foods regulations in the United States and around the world. Toxicol 2006; 221:1-3.

5. Biesalski, HK. Nutraceuticals: The Link between Nutrition and Medicine. J Toxicol Cutaneous Ocular Toxicol 2002; 21(1-2):9-30.

6. U.S. Food and Drug Administration. Questions and Answers on Dietary Supplements [Internet]. Maryland: US FDA; 2015 Apr 23.Available from: https://www.fda.gov/food/informationconsumers-using-dietary-supplements/questions-and-answersdietary-supplements (Accessed on Aug 2020)

7. Torabally NB, Rahmpoor HA. Nutraceuticals - A neutrionally functional foods - An overview. Biomed J Sci Techn Res 2019, 15(4), 1-3.

8. Eskin NAM, Tamir S. Dictionary of Nutraceuticals and Functional Foods. USA: CRC Press, Boca Raton; 2006.

9. Health claims in USA, https://www.govinfo.gov/content/pkg/FR1999-01-21/html/99-1365.htm (Accessed on Aug 2020)

10. Verma B, Popli H. Regulation of nutraceuticals in India and USA. The Pharm innovation J 2018; 7(7): 811-16.

11. US FDA How to report a problem with dietary supplements; Sep 2018; Available from: https://www.fda.gov/food/dietarysupplements/how-report-problem-dietary-supplements (Accessed on Aug 2020) 